\title{
OUTCOME OF PSEUDOANEURYSM REPAIR: A SINGLE TERTIARY CENTER EXPERIENCE IN PAHANG, MALAYSIA
}

Ahmad Faidzal Othman ${ }^{1}$, Mohamad NorHisham and Azmi Abdul Rahman

${ }^{1}$ Vascular Unit, Department Of Surgery, Kulliyah(Faculty) Of Medicine International Islamic University Malaysia / HTAA, Department Of Surgery, Kulliyah(Faculty) Of Medicine International Islamic University Malaysia/ HTAA

Presenter: Dr Ahmad Faidzal Othman, dr.faidzal@gmail.com

Introduction: Symptomatic arterial pseudoaneurysm is not an uncommon emergent vascular case presenting to a tertiary hospital. These are mainly associated with infection, iatrogenic puncture or trauma. The resultant morbidity, limb loss and mortality present a challenge to the surgeons managing these cases.

Materials and method: We retrospectively reviewed all case notes of all pseudoaneurysm repair done at our centre from Jan 2015 to May 2017.

Results: A total of 20 cases were treated surgically, with majority $16(80 \%)$ had ligation of the affected arteries. The rest were managed with reconstruction of the arteries with synthetic graft. In 7 patients (35\%), the aetiology was identified as iatrogenic intravenous drug injection. Staphylococcus aureus remained the main causative organism with Treponema Pallidum and Burkholderia pseudomallei as the other organisms identified in the cultures. Our 3 patients with graft reconstruction had long term oral antibiotics upon discharge.

Conclusion: Majority of pseudoaneurysms presented to our center results in ligation of the artery. Repair of mycotic aneurysm needs careful consideration as graft infection remains a lethal complication and no consensus is available regarding the duration of post-operative antibiotic cover. 